\title{
Stability analysis of second order pulsed Raman laser in dispersion managed systems
}

\author{
Salih K. Kalyoncu ${ }^{1}$, Shiming Gao ${ }^{1}$, En-Kuang Tien ${ }^{1}$, Yuewang Huang ${ }^{1}$, Dogukan Yildirim ${ }^{1}$, \\ Enver Adas ${ }^{1}$, Stefano Wabnitz ${ }^{2}$ and Ozdal Boyraz, \\ ${ }^{1}$ EECS Department, University of California, Irvine, 92697, USA \\ ${ }^{2}$ Dipartimento di Elettronica per l'Automazione, Università di Brescia, 25123 Brescia, Italy \\ ${ }^{3}$ EE Department, founding member, Istanbul Sehir University, Istanbul, Turkey
}

Email: oboyraz@uci.edu

\begin{abstract}
Wavelength tunable synchronous pulse sources are highly desirable for spectroscopy and optical diagnostics. The common method to generate short pulses in the fiber is the use of nonlinear induced spectral broadening which result in soliton shaping in anomalous dispersion regime. However, to generate ultra-short pulses, broadband gain mechanism is also required. In recent years, Raman fiber lasers have retrieved strong interest due to their capability of serving as pump sources in gain-flattened amplifiers for optical communication systems. The fixed-wavelength Raman lasers have been widely studied in the last years, but recently, much focus has been on the multi wavelength tunable Raman fiber lasers which generate output Stokes pulses in a broad wavelength range by so called cascaded stimulated Raman scattering. In this paper we investigate synchronous $1^{\text {st }}$ and $2^{\text {nd }}$ order pulsed Raman lasers that can achieve frequency spacing of up to $1000 \mathrm{~cm}^{-1}$ that is highly desired for CARS microscopy. In particular, analytical and numerical analysis of pulsed stability derived for Raman lasers by using dispersion managed telecom fibers and pumped by $1530 \mathrm{~nm}$ fiber lasers. We show the evolution of the 1st and 2nd order Stokes signals at the output for different pump power and SMF length (determines the net anomalous dispersion) combinations. We investigated the stability of dispersion managed synchronous Raman laser up to second order both analytically and numerically. The results show that the stable $2^{\text {nd }}$ order Raman Stokes pulses with $0.04 \mathrm{~W}$ to $0.1 \mathrm{~W}$ peak power and $2 \mathrm{ps}$ to $3.5 \mathrm{ps}$ pulse width can be achieved in dispersion managed system.
\end{abstract}

Keywords: Second order pulsed Raman laser, dispersion managed system, stability analysis, variational analysis.

\section{INTRODUCTION}

For spectroscopy and optical diagnostics, phase coherent and wavelength tunable synchronous pulse source is a great demand. The nonlinear induced spectral broadening in an anomalous dispersion regime is the widely used method to generate short pulses in the fiber by so called soliton compression [1]. Stimulated Raman scattering with a wide gain bandwidth of $\sim 6 \mathrm{THz}$ has an ability to generate femtosecond pulses. Additionally, Raman gain can be generated at any wavelength in a nonlinear medium. Up to date, the Fiber Raman Lasers (FRL) have attracted much attention for their wide spread applications such as pump sources in gain-flattened amplifiers and tunable sources for characterization of optical devices [2-3]. In particular, multi wavelength tunable fiber Raman lasers are highly desirable to generate Stokes pulses at a broad wavelength range. In chemistry and biological applications, with higher order cascaded pulsed Raman lasers with a broad frequency spacing which is highly desirable for CARS microscopy are generated [4]. In this paper, we studied the stability analysis of the $1^{\text {st }}$ and the $2^{\text {nd }}$ order pulsed Raman lasers with frequency tuning range up to $1000 \mathrm{~cm}^{-1}$. Particularly, detailed numerical analysis is conducted to investigate the stability regime of the Stokes pulses up to second order in a dispersion managed system pumped by $1530 \mathrm{~nm}$ fiber laser. Stable Stokes pulses are generated with respect to different pump power and single mode fiber (SMF) length that consists of dispersion managed (DM) system to form the cavity. Variational analysis is also derived to analytically analyze the steady state dynamics of the Stokes pulses inside the cavity and to predict the output pulse parameter by solving ordinary differential equations with a periodic boundary conditions which seems an alternative to time consuming numerical simulations. The analytical results are compared with simulation results to show good agreement with the derived equations. The results illustrate that the stable $2^{\text {nd }}$ order Raman Stokes pulses with $0.04 \mathrm{~mW}$ to $0.1 \mathrm{~W}$ peak power and $2 \mathrm{ps}$ to $3.5 \mathrm{ps}$ pulse width can be achieved in DM system.

Nonlinear Optics and Applications V, edited by Mario Bertolotti, Proc. of SPIE Vol. 8071 $807116 \cdot$ ? 2011 SPIE $\cdot$ CCC code: $0277-786 X / 11 / \$ 18 \cdot$ doi: $10.1117 / 12.889716$ 


\section{SIMULATION SETUP and ANALYTICAL EQUATIONS}

To generate stable pulsed lasers by Raman process, dispersion management of pump, first order and second order pulses is the utmost important parameter. Most of the commercial DSFs and SMFs have high dispersion values and slopes, and hence it is not possible to maintain a reasonable walk-off (overlapping of pulses) through the propagation. To eliminate walk off and perform stability analysis following experimental setup is being investigated, Figure 1a.
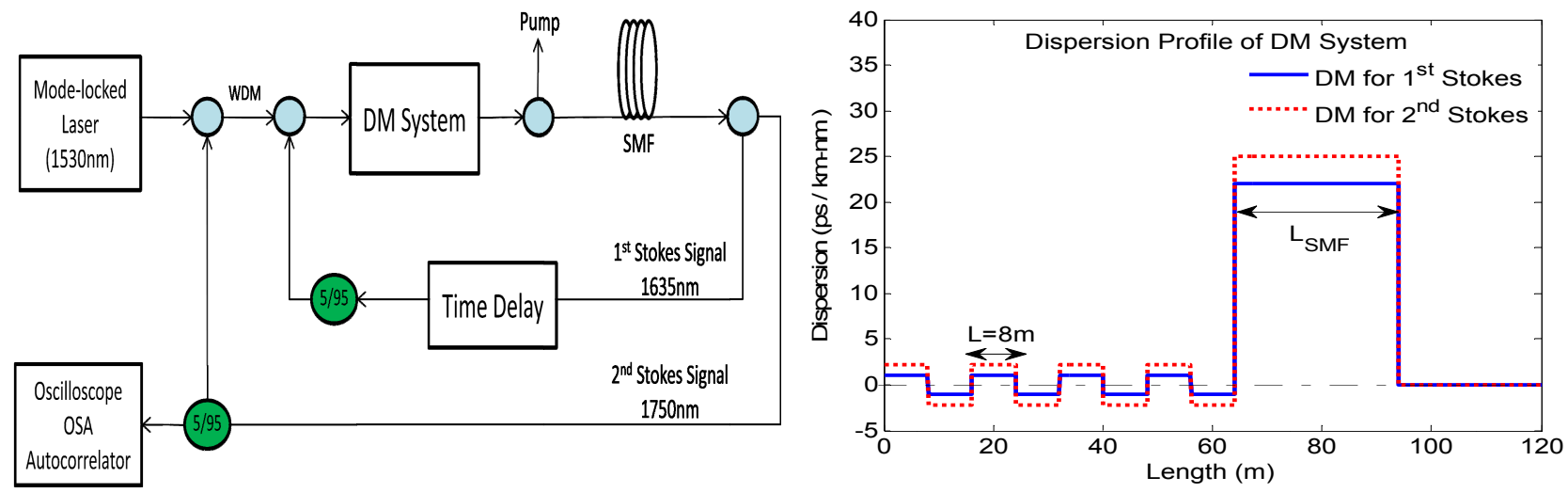

Figure 1. Designed set up (1a) and the dispersion managed system for each Stokes pulses (1b).

System is pumped by $1530 \mathrm{~nm}$ Mode Locked Laser with 10ps pulse width. The laser cavity is designed as proposed in [7] that mainly consists of two cascaded sections, the dispersion managed (DM) system as the gain mechanism and the single mode fiber (SMF) for pulse shaping. Dispersion management is formed by cascading highly nonlinear fiber (HNLF) pieces which has a nearly zero dispersion and dispersion slope in a periodic configuration. In particular, we restrict our analysis for commercially available fibers from different vendors. The characteristics of the HNLF's and the single mode fiber (SMF) used in the simulations are summarized in Table 1.

Table 1. The characteristics of the fibers used in the simulations [6].

\begin{tabular}{|c|c|c|c|}
\hline & HNLF (+) slope & HNLF (-) slope & SMF \\
\hline Fiber loss $(\alpha)$ & $0.9 \mathrm{~dB} / \mathrm{km}$ & $0.9 \mathrm{~dB} / \mathrm{km}$ & $0.2 \mathrm{~dB} / \mathrm{km}$ \\
\hline Nonlinearity coefficient $(\gamma)$ & $11.5(1 / \mathrm{W}-\mathrm{km})$ & $11.5(1 / \mathrm{W}-\mathrm{km})$ & $2(1 / \mathrm{W}-\mathrm{km})$ \\
\hline $\mathrm{A}_{\text {eff }}$ & $11.7\left(\mu \mathrm{m}^{2}\right)$ & $11.7\left(\mu \mathrm{m}^{2}\right)$ & $50\left(\mu \mathrm{m}^{2}\right)$ \\
\hline Raman coefficient $\left(\mathrm{g}_{\mathrm{R}}\right)$ & $3.4(1 / \mathrm{W}-\mathrm{km})$ & $3.4(1 / \mathrm{W}-\mathrm{km})$ & $0.8(1 / \mathrm{W}-\mathrm{km})$ \\
\hline Zero dispersion $\left(\lambda_{0}\right) /$ slope & $1530 \mathrm{~nm} / 0.01\left(\mathrm{ps} / \mathrm{nm}^{2} \mathrm{~km}\right)$ & $1530 \mathrm{~nm} /-0.01\left(\mathrm{ps} / \mathrm{nm}^{2} \mathrm{~km}\right)$ & $1310 \mathrm{~nm} / .076\left(\mathrm{ps} / \mathrm{nm}^{2} \mathrm{~km}\right)$ \\
\hline Length & $\mathrm{L}=8 \mathrm{~m}(4 \mathrm{section})$ & $\mathrm{L}=8 \mathrm{~m}(4 \mathrm{section})$ & $\mathrm{L}=20 \mathrm{~m}-100 \mathrm{~m}$ \\
\hline
\end{tabular}

In order to raise the net dispersion to anomalous regime, SMF is cascaded with the dispersion managed system to generate stable soliton like pulses. Thus, each round trip can be assumed as a period of the modified dispersion managed system having net anomalous dispersion. Figure $1 \mathrm{~b}$ illustrates the dispersion profile for each Stokes order in fibers that satisfies the described design criteria. The overlapping of Stokes pulses at the beginning is satisfied by inserting a stretcher (delay line) in one of the ring cavities to match the pump repetition rate. 
The propagation of Stokes pulses at steady state can be modeled by using variational analysis method which assumes the signal maintains its shape even though its power, pulse width and chirp changes in a continuous manner and remains same periodically after each round trip. The well known equations describing the evolution of single pulse parameters in variational method [5] can be modified by taking signal to signal interactions into consideration such as Raman amplification, cross phase modulation (XPM) and walk off as a perturbation in the system. In particular, the physical effects of XPM and the walk off on the chirp evolution, pump depletion, and gain reduction become more dominant [8]. The modified analytical equations for the pulse parameters, power $(\mathrm{P})$, pulse width $(\mathrm{T})$, chirp $(\mathrm{C})$ and the temporal position $(\mathrm{t})$ assuming all the pulses having Gaussian shape are:

$$
\begin{aligned}
& \frac{d P_{p}}{d z}=-\alpha P_{p}-g_{R} \frac{\lambda_{s 1}}{\lambda_{p}} P_{1} \exp \left[-\left(\frac{t_{1}}{T_{1}}\right)^{2}\right] P_{p}+\left(\frac{t_{p}}{T_{p}}\right) \frac{d}{d z}\left(\frac{t_{p}}{T_{p}}\right) \\
& \frac{d}{d z}\left(\frac{t_{p}}{T_{p}^{2}}\right)=w_{1} \\
& \frac{d P_{1}}{d z}=-\alpha P_{1}+g_{R} P_{p} \exp \left[-\left(\frac{t_{p}}{T_{1}}\right)^{2}\right] P_{1}-g_{R} \frac{\lambda_{s 2}}{\lambda_{s 1}} P_{2} \exp \left[-\left(\frac{t_{2}}{T_{2}}\right)^{2}\right] P_{1}-\frac{P_{1}}{T_{1}} \frac{d T_{1}}{d z}+\left(\frac{t_{1}}{T_{1}}\right) \frac{d}{d z}\left(\frac{t_{1}}{T_{1}}\right) \\
& \frac{d T_{1}}{d z}=\frac{\beta_{21} C_{1}}{T_{1}}-\frac{g_{R} T_{1}^{3}}{2}\left(\frac{P_{p}}{T_{p}^{2}}-\frac{\lambda_{s 2}}{\lambda_{s 1}} \frac{P_{2}}{T_{2}^{2}}\right) \\
& \frac{d C_{1}}{d z}=\left(1+C_{1}^{2}\right) \frac{\beta_{21}}{T_{1}^{2}}+\frac{\gamma}{\sqrt{2}}\left[P_{1}+2 P_{p}\left(\frac{T_{1}}{T_{p}}\right)^{2}+2 P_{2}\left(\frac{T_{1}}{T_{2}}\right)^{2}\right]-g_{R} T_{1}^{2} C\left(\frac{P_{p}}{T_{p}^{2}}-\frac{\lambda_{s 2}}{\lambda_{s 1}} \frac{P_{2}}{T_{2}^{2}}\right) \\
& \frac{d}{d z}\left(\frac{t_{1}}{T_{1}^{2}}\right)=w_{2}+g_{R} t_{1}\left(\frac{P_{p}}{T_{p}^{2}}-\frac{\lambda_{s 2}}{\lambda_{s 1}} \frac{P_{2}}{T_{2}^{2}}\right) \\
& \frac{d P_{2}}{d z}=-\alpha P_{2}+g_{R} P_{1} \exp \left[-\left(\frac{t_{1}}{T_{1}}\right)^{2}\right] P_{2}-\frac{P_{2}}{T_{2}} \frac{d T_{2}}{d z}+\left(\frac{t_{2}}{T_{2}}\right) \frac{d}{d z}\left(\frac{t_{2}}{T_{2}}\right) \\
& \frac{d T_{2}}{d z}=\frac{\beta_{22} C_{2}}{T_{2}}-\frac{g_{R} T_{2}^{3}}{2} \frac{P_{1}}{T_{1}^{2}} \\
& \frac{d C_{2}}{d z}=\left(1+C_{2}^{2}\right) \frac{\beta_{22}}{T_{2}^{2}}+\frac{\gamma}{\sqrt{2}}\left[P_{2}+2 P_{p}\left(\frac{T_{2}}{T_{p}}\right)^{2}+2 P_{1}\left(\frac{T_{2}}{T_{1}}\right)^{2}\right]-g_{R} T_{2}^{2} C\left(\frac{P_{1}}{T_{1}^{2}}\right) \\
& \frac{d}{d z}\left(\frac{t_{2}}{T_{2}^{2}}\right)=g_{R} t_{2}\left(\frac{P_{1}}{T_{1}^{2}}\right)
\end{aligned}
$$

where $w_{1}=\int_{\lambda_{p}}^{\lambda_{1}} D(\lambda) d \lambda \cong \frac{\left(D_{p}+D_{1}\right)}{2}\left(\lambda_{1}-\lambda_{p}\right)$ and $w_{2}=\int_{\lambda_{1}}^{\lambda_{2}} D(\lambda) d \lambda \cong \frac{\left(D_{2}+D_{1}\right)}{2}\left(\lambda_{2}-\lambda_{1}\right)$. Here the subscripts $\mathrm{j}=\mathrm{p}, 1,2$ correspond to pump, $1^{\text {st }}$ Stokes and $2^{\text {nd }}$ Stokes signals respectively. In addition, $\alpha$ is the fiber loss 
$\left(\mathrm{km}^{-1}\right), \mathrm{g}_{\mathrm{R}}$ is the Raman gain coefficient $\left(\mathrm{W}^{-1} \mathrm{~km}^{-1}\right), \gamma$ is the nonlinearity coefficient $\left(\mathrm{W}^{-1} \mathrm{~km}^{-1}\right), \mathrm{D}$ is the dispersion value $(\mathrm{ps} / \mathrm{nm}-\mathrm{km}), \beta_{2}$ is the group velocity dispersion parameter $\left(\mathrm{ps}^{2} / \mathrm{km}\right)$ and $\mathrm{w}_{1,2}$ stands for walk of times between pump- $1^{\text {st }}$ Stokes and $1^{\text {st }}-2^{\text {nd }}$ Stokes pulses.

\section{SIMULATION RESULTS and STABILITY ANALYSIS}

The main objective of the analysis is to determine if the output Stokes pulses converge to dispersion managed solitons at steady state. Split Step Fourier Method (SSFM) is being used to solve the coupled NLS equations. The $1^{\text {st }}$ and $2^{\text {nd }}$ order Stokes signals are obtained at the output for different pump power and SMF length (determines the net anomalous dispersion) combinations. In Figure 2, the output Stokes pulses and their peak power evolution are shown as an example of transient analysis. The envelope of the output Stokes pulses is also characterized by comparing with a Gaussian pulse with the same pulse parameters.
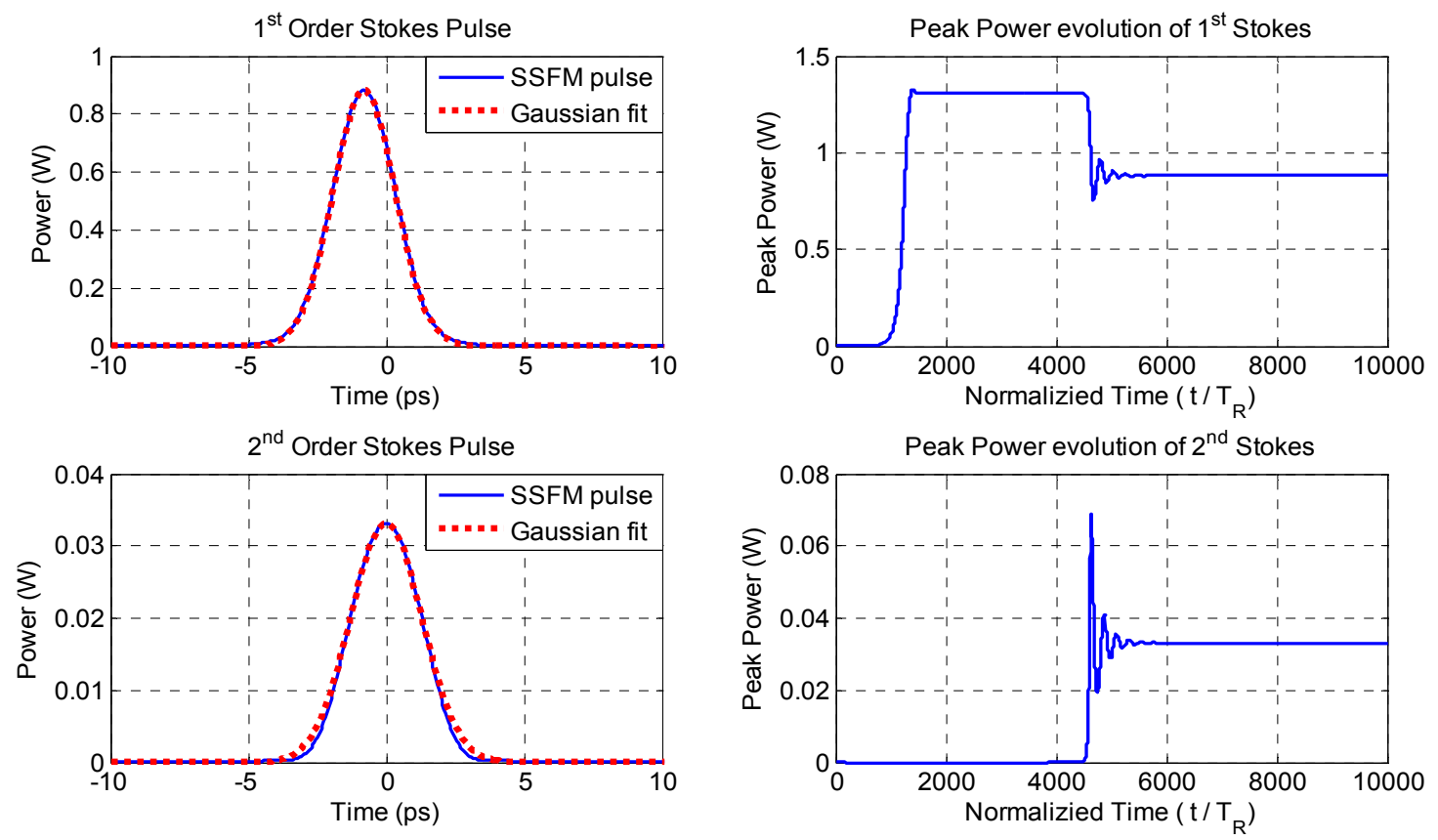

Figure 2. The output Stokes pulses (2a) with Gaussian fitting and the evolution of their peak powers $(2 \mathrm{~b})$ at $\mathrm{L}_{\mathrm{SMF}}=50 \mathrm{~m}$ and $\mathrm{P}_{\text {pump }}=0.72 \mathrm{~W} . \mathrm{T}_{\mathrm{RT}}$ is the round trip time of the cavity.

The intra cavity pulse dynamics of the Stokes pulses are also investigated by extracting the evolution of pulse parameters through the single pass. Figure 3 illustrates the evolution of peak power and the pulse width (FWHM) of the Stokes pulses at steady state. The dynamics of the pulse parameters of the Stokes pulses has similar characteristics. Through the dispersion managed section of the cavity where all the Raman interactions occur, the gain and the nonlinear effects such as single phase modulation (SPM) and the cross phase modulation (XPM) are the dominant terms. Thus, the Stokes pulse are amplified and positively chirped though the dispersion managed (DM) fibers. Because the dispersion length $\left(\mathrm{L}_{\mathrm{D}}=\mathrm{T}_{0}^{2} /\left|\beta_{2}\right|\right)$ through the individual highly nonlinear fibers (HNLF) is much more than the amplification $\left(\mathrm{L}_{\mathrm{A}}=\left(\mathrm{g}_{\mathrm{R}}|\mathrm{A}|^{2}\right)^{-1}\right)$ and nonlinear lengths $\left(\mathrm{L}_{\mathrm{NL}}=\left(\gamma|\mathrm{A}|^{2}\right)^{-1}\right)$ and due to net zero dispersion of the DM system, linear dispersive effects become negligible as seen by the pulse width. However, in the second section of the cavity (standard SMF), since the pulses become separate due the walk off, there is no pulse to pulse interaction. The group velocity dispersion (GVD) is the dominant effect though the SMF. Since the pulses becomes highly positively chirped in the DM section of the cavity due to nonlinear phase, the Stokes pulses first compress and then broaden as seen by the dynamic of peak power and pulse width. 

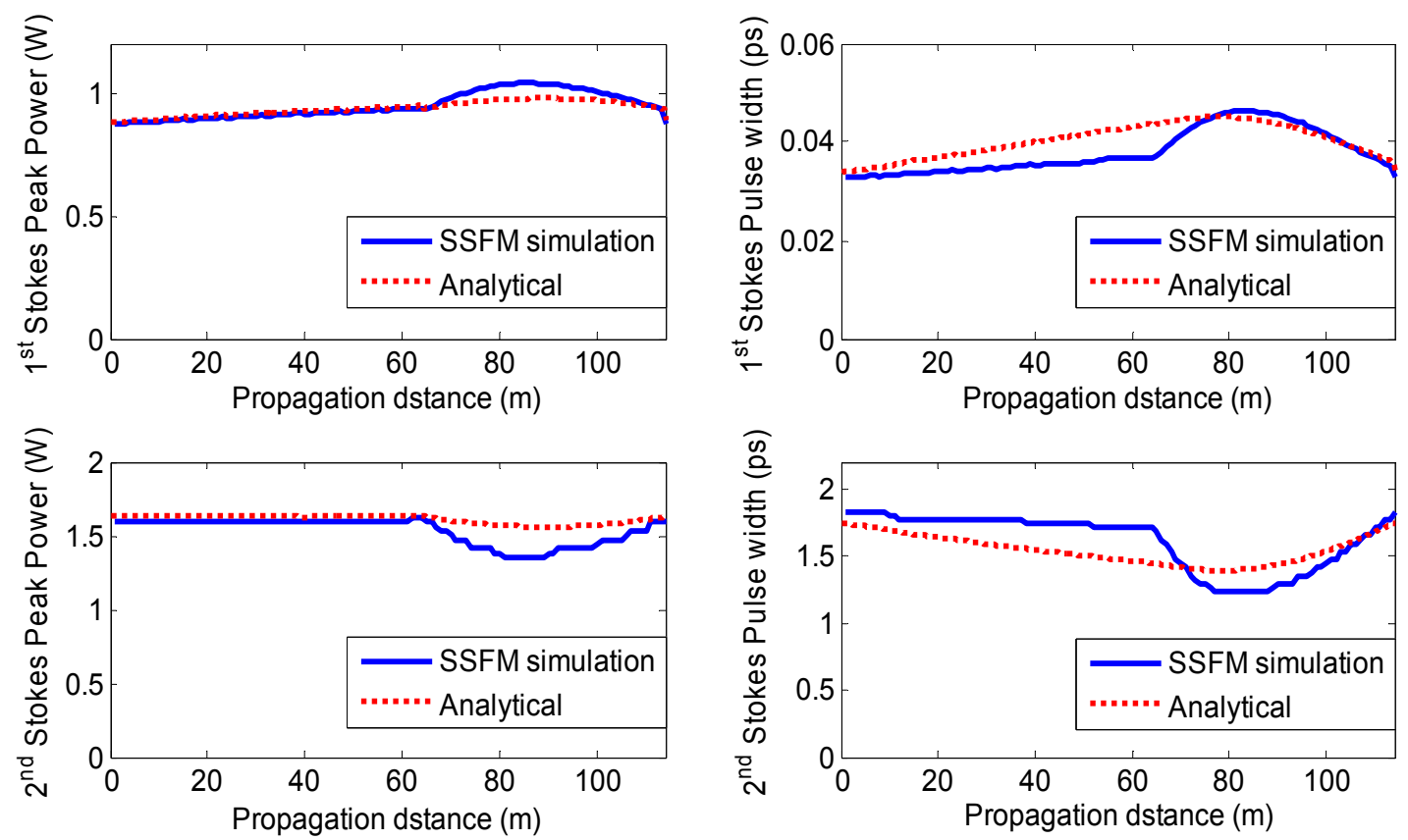

Figure 3. The intra-cavity dynamics of the steady state pulse parameters, peak power and pulse width of $1^{\text {st }}$ order Stokes $(3 \mathrm{a})$ and $2^{\text {nd }}$ order Stokes (3b) at $\mathrm{L}_{\mathrm{SMF}}=50 \mathrm{~m}$ and $\mathrm{P}_{\mathrm{pump}}=0.72 \mathrm{~W}$ are shown. The analytical and simulation results are compared.
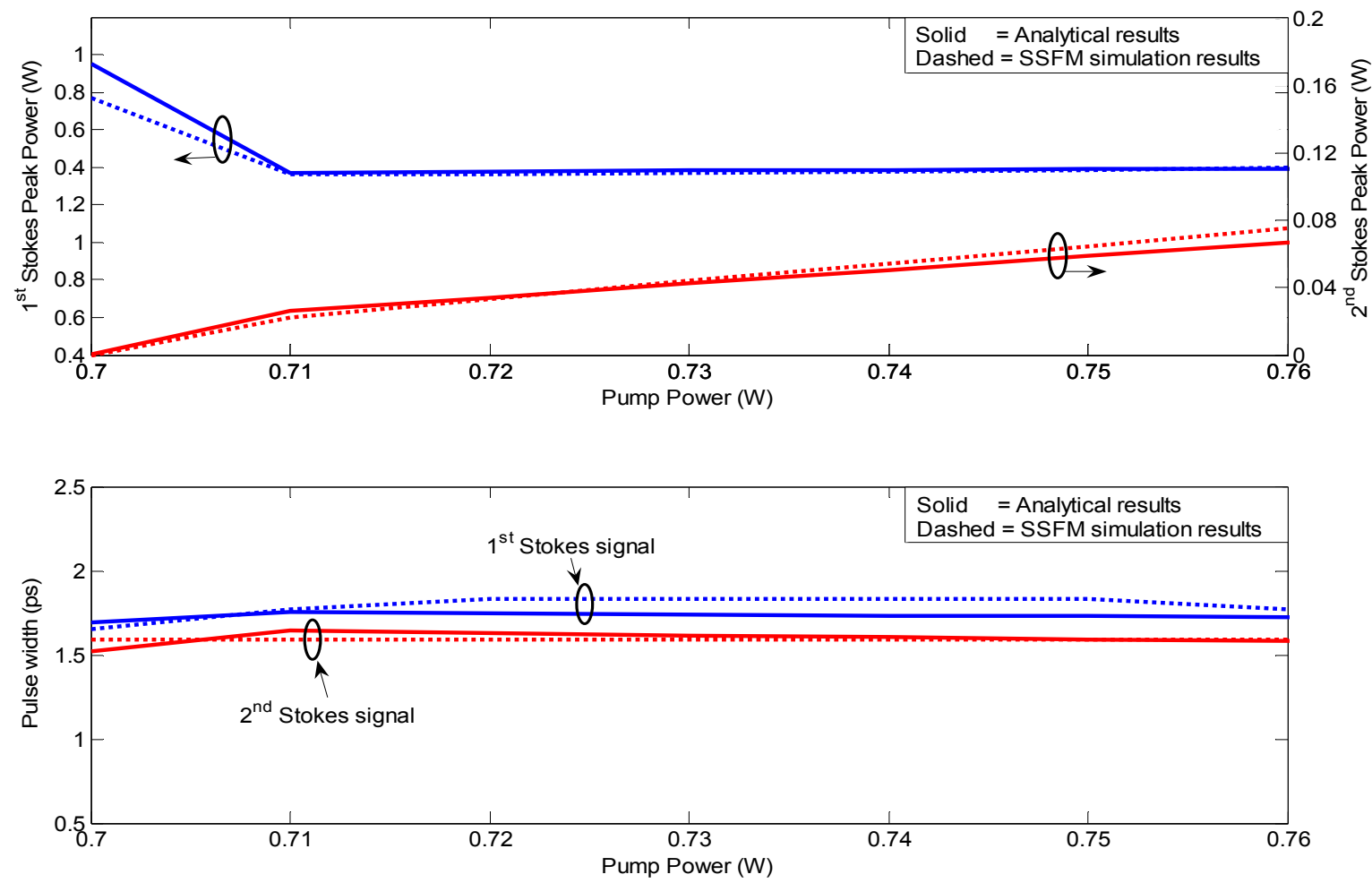

Figure 4. Steady state peak power (4a) and pulse width (4b) of the Stokes pulses with respect to pump power are shown. The correctness of analytical derivations is also illustrated in the figure by comparing with the simulation results. 
Analytically derived pulse parameters such as peak power and pulse width of the $1^{\text {st }}$ and the $2^{\text {nd }}$ order Stokes at steady state with respect to different pump powers are also illustrated in Figure 4 with numerical results. The intra cavity dynamics of these parameters are analyzed by assuming the steady state values found by solving the differential equations at any point inside the cavity as the initial condition and integrating the equations for one round trip [9]. The analytical and numerical results are shown Figure 3 for specific SMF length and pump power $\left(\mathrm{L}_{\mathrm{SMF}}=50 \mathrm{~m}\right.$ and $\mathrm{P}_{\text {pump }}=0.72 \mathrm{~W}$ ). The agreement with the numerical results obtained by SSFM verifies the validity of our variational analysis.

To investigate the stable regime of the output Stokes pulses, system is deeply analyzed by changing both the length of the SMF from $20 \mathrm{~m}$ to $100 \mathrm{~m}$ and for each case tuning the pump peak from $0.7 \mathrm{~W}$ to $0.76 \mathrm{~W}$. The results are shown in the following contour plots indicating the peak power and pulse widths of $1^{\text {st }}$ and $2^{\text {nd }}$ order Stokes pulses.
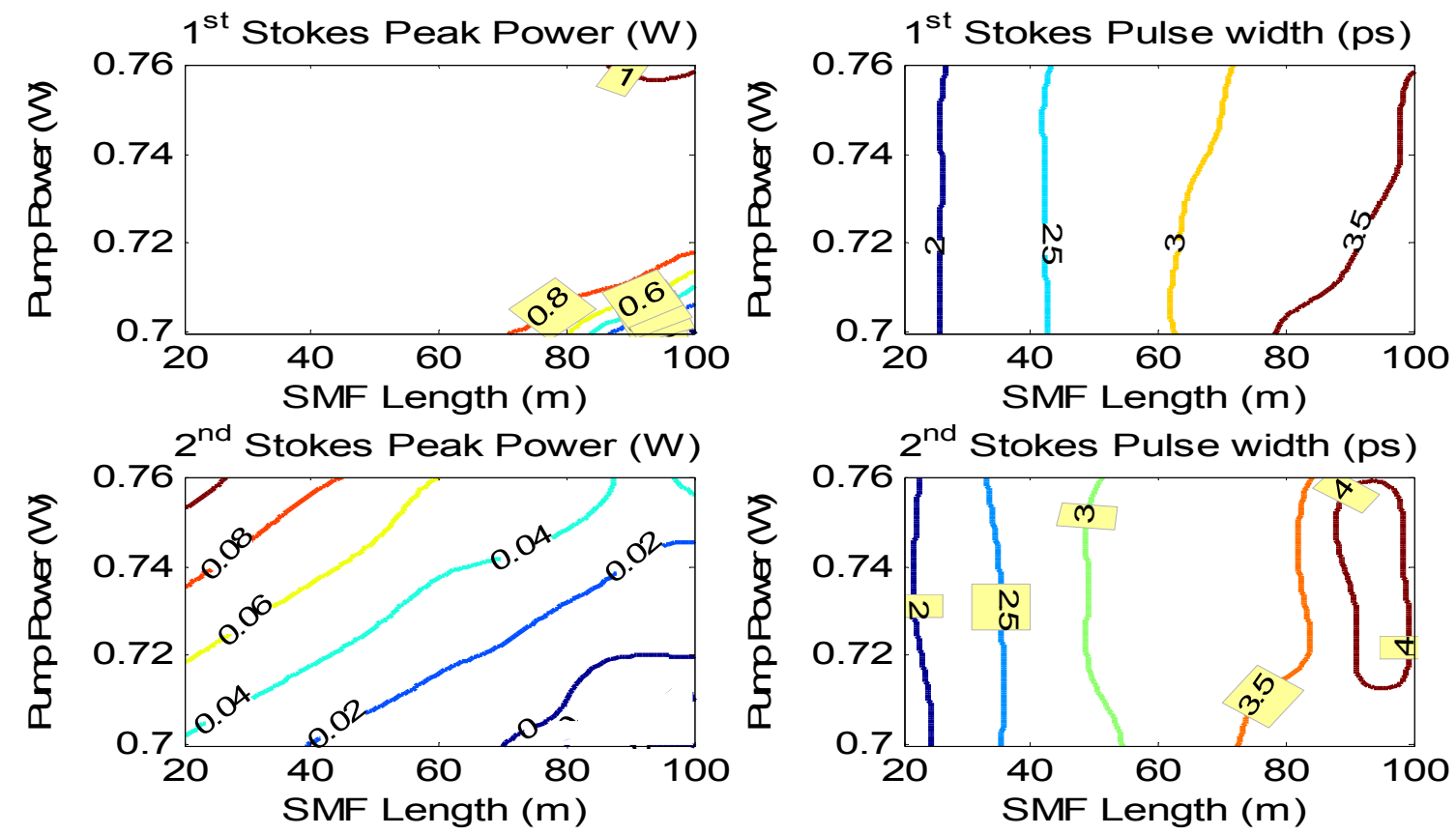

Figure 5. Contour plots for the peak power and pulse width of the $1^{\text {st }}$ and $2^{\text {nd }}$ order Stokes pulses due to the SMF length and pump power.

Figure 5 illustrates the peak powers and pulse widths at which the output Stokes pulses converge at steady state. At low pump powers which are very close to threshold only the $1^{\text {st }}$ order Stokes can be generated. When the pump power is increased, $1^{\text {st }}$ order Stokes signal starts to build up in the system and converge. If the final peak power of the $1^{\text {st }}$ Stokes signal reaches the threshold for $2^{\text {nd }}$ Stokes signal, it generates the $2^{\text {nd }}$ Stokes pulse. At high pump powers due to imbalance between strong nonlinearity and weak dispersion, $1^{\text {st }}$ Stokes signal starts to break up and system becomes unstable. The robustness to nonlinearity increases proportionally with net dispersion.

\section{CONCLUSION}

We investigated the stability of dispersion managed synchronous Raman laser up to second order both analytically and numerically. The variational analysis is conducted to derive the differential equations which explain the intra cavity dynamics of Stokes pulses in terms of their pulse parameters. The coupled differential equations with a periodic boundary conditions are solved to obtain the output Stokes pulse parameters at steady state. The analytically and numerically derived pulse parameters of the Stokes pulses are compared to illustrate the correctness of the analytical analysis. The results show that the stable $2^{\text {nd }}$ order Raman Stokes pulses with $0.04 \mathrm{~W}$ to $0.1 \mathrm{~W}$ peak power and $2 \mathrm{ps}$ to 
3.5ps pulse width can be achieved in DM system. Results can be further improved by optimizing the parameters of the dispersion management.

\section{ACKNOWLEDGEMENT}

This work is supported by DARPA RADER Program and EU grant PIRG07-GA-2010-268370.

\section{REFERENCES}

[1] C.J.S. Matos, S.V. Popov, and J.R. Taylor, "Short-pulse, all-fiber, Raman laser with dispersion compensation in a holey fiber" Opt. Lett. Vol. 28, No. 20 (2003).

[2] G H. Renner, S. Cierullies, M. Krause, "Scaling rules for Raman fiber lasers" OFC, Atlanta, USA (Paper MF25), (2003).

[3] M. Krause, H. Renner, “Theory and design of double-cavity Raman fiber lasers” J. Lightwave Technology, Vol. 23, No. 8 (2005).

[4] S. Cierullies, H. Renner, E. Brinkmeyer, "Numerical optimization of multi-wavelength and cascaded Raman fiber lasers" Opt. Commun. 217(1-6), pp. 233-238 (2003).

[5] G.P. Agrawal, [Nonlinear Fiber Optics $4^{\text {th }}$ edition], Academic Press (2009).

[6] OFS Specialty Photonics Division, http://ofscatalog.specialtyphotonics.com

[7] H.A. Haus and M. Nakazawa, "Theory of the fiber Raman soliton laser", J. Opt. Soc. Am. B/Vol. 4, No.5, (May/1987).

[8] A. Hook, D. Anderson, and M. Lisak, "Effects of cross-phase modulation and pump depletion on Stokes pulse dynamics in stimulated Raman scattering", J. Opt. Soc. Am. B, Vol. 6, No.10, (Oct/1989).

[9] Cristian Antonelli, Jeff Chen, Franz X Kartner, "Intracavity pulse dynamics and stability for passively mode-locked lasers", Optics Express, Vol. 15, No.10, (May/2007). 